Recepción: 13 / 07 / 2018

Aceptación: 21 / 09 / 2018

Publicación: 05 / 10 / 2018

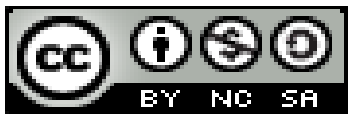

Ciencias sociales y políticas

Artículo original

\title{
El fortalecimiento de la resiliencia en la cultura de riesgos de Manta
}

\author{
Strengthening resilience in the risk culture of Manta
}

Fortalecendo a resiliência na cultura de risco da Manta

Wagner O. Villafuerte-Muñoz ${ }^{\text {I }}$

wagnermatador38@gmail.com

Correspondencia: wagnermatador38@gmail.com

I Diploma Superior en Gestión Directiva, Magíster en Enseñanza del Idioma Ingles, Licenciado en Ciencias de la Educación Mención en Inglés, Docente de la Universidad Laica Eloy Alfaro de Manabí, Manta, Ecuador. 


\section{Resumen}

El escenario geográfico, donde se desenvuelve el hábitat de los ecuatorianos, debe ser analizado detenidamente, considerando todos los factores internos y externos que intervienen, que inciden en el normal desenvolvimiento en todos los aspectos, a fin de adoptar comportamientos necesarios para poder enfrentar eventualidades que podrían ocurrir, ocasionando desastres frente a todo tipo de riesgos. También debe de considerarse la correlación de las fallas geológicas como el movimiento de las placas tectónicas, el ser parte del Cinturón de Fuego, la cordillera de los Andes, volcanes activos en el continente y en el océano, entre otros. Las ciudades deben determinar la manera de adaptarse, sobrevivir y reincorporarse de acuerdo a las circunstancias de adversidad que pudieren atravesar, considerando los desastres como una oportunidad de cambio, ambientándose a las dificultades y limitaciones, las que pudieran ser originadas por el poder de la naturaleza o por el ser humano. Según el portal ONU Hábitat, las ciudades resilientes, son aquellas que están preparadas para el cambio y cuentan con medidas adecuadas para recuperarse de una crisis, este tipo de ciudades promueven el bienestar de los habitantes con medidas que beneficien de manera colectiva su estructura o funcionamiento. La resiliencia va más allá de la reconstrucción y el manejo de la emergencia, donde muchas actividades deben ser consideras como estrategia para enfrentar los resultados de las catástrofes. Dentro de la cultura de riesgos, podríamos considerar el Turismo, actividad que involucra la participación de los mantenses. Son múltiples las estrategias que podrían utilizarse a fin de fortalecer el levantamiento en todos los aspectos de la ciudad de Manta.

Palabras clave: resiliencia; cultura de riesgos; turismo; catástrofes; placas tectónicas.

\section{Abstract}

The geographical scenery, where it operates the habitat of the Ecuadorians, must be carefully analyzed, considering all the internal and external factors involved that affect the normal development in all aspects, taking behavior necessary, before contingencies that may occur, causing natural disasters all kinds of risks. The Ecuadorian soil is of high seismicity according to the statistical data of earthquakes and the correlation with the geological faults such as the movement of the tectonic plates, being part of the Belt of Fire, the Andes mountain range, among others. Cities must determine how to adapt and reincorporate their way of living according to the circumstances they traverse, considering as an opportunity for change to evolve and transform the 
cities that have been, as results of Disasters. According to the UN Habitat portal, resilient cities, they are those that are prepared for the change and are provided with measurements adapted to recover of a crisis, since this type of cities promotes the well-being of the inhabitants with measurements who benefit in a collective way its structure or functioning. The resilience goes beyond the reconstruction and the handling of the emergency, where the tourist activity redeems a very important roll in the search of this well-being, foodstuff or motorcars, turning into one of the main actors of the international trade, and generating diversification and competition. The applied methodology was qualitative of descriptive character, deciding that the Marketing Holístico applied to the commercial development, he will allow to study the needs of the consumers, so that of sustainable form and sustentable.

Keywords: Resilience; risk culture; tourism; catastrophes; tectonic plates.

\section{Resumo}

A configuração geográfica, onde o habitat dos equatorianos opera, deve ser analisada com cuidado, considerando todos os fatores internos e externos envolvidos, afetando o desenvolvimento normal em todos os aspectos, a adotar comportamentos necessários para lidar com eventualidades poderia acontecer, causando desastres em face de todos os tipos de riscos. A correlação de falhas geológicas também deve ser considerada, como o movimento de placas tectônicas, fazendo parte do Cinturão do Fogo, a Cordilheira dos Andes, vulcões ativos no continente e no oceano, entre outros. As cidades devem determinar a maneira de se adaptar, sobreviver e reincorporar de acordo com as circunstâncias da adversidade que poderiam passar, considerando os desastres como uma oportunidade de mudança, fixando-se às dificuldades e limitações, que poderiam ser causadas pelo poder da natureza. ou pelo ser humano. Segundo o portal da ONU Habitat, cidades resilientes são aquelas que estão preparadas para a mudança e têm medidas adequadas para se recuperar de uma crise, essas cidades promovem o bem-estar dos habitantes com medidas que beneficiam coletivamente sua estrutura ou funcionamento. A resiliência vai além da reconstrução e gestão da emergência, onde muitas atividades devem ser consideradas como uma estratégia para enfrentar os resultados das catástrofes. Dentro da cultura de riscos, poderíamos considerar o Turismo, uma atividade que envolve a participação do povo de Mântua. Existem várias estratégias que podem ser usadas para fortalecer a insurreição em todos os aspectos da cidade de Manta. 
Palavras chave: Resiliência; cultura de risco; turismo; catástrofes; placas tectônicas.

\section{Introducción}

\section{Situación problemática}

A pesar de los avances y desarrollos de la tecnología, aun no existen equipos creados por el ser humano, que permitan establecer un sistema de Alarma Temprana, que proporcione el tiempo suficiente para lograr una evacuación correcta y segura, preservando la vida del ser humano; en tal virtud es menester fortalecer acciones en la cultura de riesgos, a fin de enfrentar, sobrevivir y desarrollar con eventual normalidad ante situaciones catastróficas, con una elevada RESILIENCIA en el cantón de San Pablo de Manta.

Dentro del análisis para considerar y establecer factores incidentes y determinantes, es necesario mencionar que de acuerdo a la publicación realizada por el Diario El Comercio del 26 de agosto del 2014, a las 14:25 indica que nuestro país Ecuador está asentado sobre tres sistemas de fallas geológicas, como producto de la subducción de la Placa de Nazca en el océano, con la Placa Sudamericana, siendo necesaria la reflexión del escenario mantense, ya que debemos concientizarnos del tipo de suelo elevadamente sísmico y la correlación existente con las fallas geológicas a nivel mundial, estas se encuentran demarcadas en diferentes lugares geográficos en todos los continentes, incluyendo lo que acontece en los océanos, lo que invita a la reflexión y búsqueda de soluciones estratégicas a nivel gubernamental, social, grupal e individual, minimizando las catastróficas consecuencias que podría generar un desastre natural o creado por el hombre. Es importante contar con acciones que brinden soluciones durante los tres tiempos de un desastre: antes, durante y después a los hechos.

Lo impredecible que resulta el poder determinar el momento en que se desarrollen los desastres naturales y sus temibles consecuencias, crea escenarios difíciles de imaginar. Existen equipos de avanzada tecnología que tan solo facilitan segundos de anticipación ante la ocurrencia de un desastre (Terremoto-Tsunami). Siendo trascendental crear una planificación estratégica, que abarquen planes de contingencia y evacuación ante desastres, debiendo realizar permanentes simulacros de Evacuación, fortaleciendo la Resiliencia. 
Las graves consecuencias que podrían generar los resultados de los desastres, crean momentos limitantes para el normal desenvolvimiento de los ciudadanos. Las ciudades construidas sobre fallas geológicas por lo general carecen de una planificación adecuada, siendo más vulnerables y riesgosas, por lo tanto la ayuda del sector gubernamental así como el de las empresas privadas representan un factor clave en la generación de planes de acción y de cooperación mutua que permitan transformarlas y convertirlas en ciudades más seguras debido a que éstas constituyen el motor de desarrollo de un país, y los desastres ejercen presión sobre la población en su bienestar y proyección.

El desarrollo de la resiliencia y la identificación de buenas prácticas y herramientas, ayudan a la creación de estrategias urbanas para lograr un desarrollo sostenible y sustentable; pues existe una elevada probabilidad de que los eventos naturales incrementan los riesgos de mayor magnitud, siendo responsabilidad de los gobiernos locales dar respuestas ante dichos desastres; no obstante, las capacidades limitadas con las que cuentan estos organismos no les permite anticipar, gestionar y reducir los riesgos, por lo que se crea la necesidad de verificar las asignaciones de recursos que proporcione e incrementen dichas capacidades a fin de que puedan dar solución a los problemas, imprevistos y desafíos en función de los riesgos generados.

Los países en vías de desarrollo requieren de gobernantes responsables con su entorno y sociedad, de profesionales capacitados en áreas estratégicas, competentes para ser parte del equipo que haga frente a eventualidades catastróficas, motivados por nuevas demandas y necesidades que en su gran mayoría no son satisfechas por las instituciones gubernamentales, requiriendo de un cambio de actitud e inclusión de valores para que comprendan tensiones y problemas con un rol protagónico en el progreso económico de la región donde la globalización implica un desarrollo científico, tecnológico e innovador, que permita un crecimiento inclusivo y sostenible para los ciudadanos, es decir que es preponderante la participación gubernamental y de cada uno de los ciudadanos mantenses.

Múltiples son las actividades que podrían desarrollarse posterior a un desastre en un escenario con elevado grado de riesgos, una de ellas es la que el presente artículo plantea, considerando la ubicación geográfica del cantón San Pablo de Manta, explotando las bondades naturales existentes como su cercanía al mar con un aporte exclusivo en la economía de nuestro país, donde 
existe gran afluencia de turistas provenientes de diferentes continentes a través de los cruceros, por ser Manta uno de los puertos hondos y principales del Ecuador, lo cual ha permitido incrementar un importante crecimiento en las actividades económicas que se ven afectadas después de un desastre, sin embargo, estratégicamente ha experimentado un continuo crecimiento y una profunda diversificación, hasta convertirse en uno de los sectores económicos que crecen con mayor rapidez en el mundo.

El turismo se transforma en un elemento altamente estratégico, tanto a nivel local como mundial, guardando una estrecha relación con el desarrollo socioeconómico de nuestra nación, siendo la cuidad de Manta pilar fundamental en la economía del país a través del turismo, estas acciones están inmersas en el fortalecimiento de la resiliencia de la ciudad de Manta, esto se ha convertido en una dinámica, ya que es el motor que genera divisas y permite el resurgimiento ante situaciones de desastre.

Esta actividad económica necesita apoyarse en la disciplina del marketing; transformándose en una doctrina de filosofía empresarial, sirviendo de guía a todas las organizaciones, desde lo holístico donde analiza procesos y reconoce el alcance e interdependencia de cada uno de sus efectos; por lo tanto, es necesario tener una visión amplia e integrada que involucre a todas instituciones, organizaciones, personas, espacios y lugares que tienen que ver con la empresa, como: empleados, clientes, empresas competidoras, sociedad entre otros. Esta orientación tiene como clave la determinación de las necesidades y valores de la población objetivo, con el fin de conseguir el deseado nivel de satisfacción. Por eso, en los actuales momentos, las organizaciones son más sensibles a las necesidades, preferencias y expectativas de sus clientes o usuarios, establecen una relación entre el entorno externo e interno, alinean la cultura organizacional, las tecnologías de información y comunicación, y el capital humano. Mediante el trabajo en equipo agregan valor a entidad, al servicio que brinda o al producto que venden; acercándonos al concepto de marketing holístico, siendo necesario conocer la real y verdadera definición de estos conceptos provenientes de aula, textos y demás documentos, a fin de que sean prácticos y aplicativos en beneficios de la resiliencia en Manta para enfrentar adversidades.

Esta investigación, tiene el propósito de que los lectores comprendan la importancia de la resiliencia antes y después de un desastre. Provee una relación de la disciplina del marketing 
holístico y la resiliencia en la industria turística, como factor fundamental para generar negocios y oportunidades, innovar procesos y aplicar estrategias que impulsen el desarrollo post desastre del 16A. La resiliencia incluye conductas, pensamientos y acciones que pueden ser aprendidas y desarrolladas por cualquier persona; ejemplo de ello, las respuestas de las personas a los ataques terroristas del 11 de septiembre del 2001 ocurrido en los Estados Unidos, quienes demostraron mucha tristeza y sufrimiento, pero a la vez superaron adversidades y obstáculos, volviendo a levantarse entre los escombros.

\section{Problema}

¿Ante las fallas geológicas de Manabí y estadísticas alarmantes de movimientos telúricos en el Ecuador, existe la elevada preocupación de la ciudadanía, que limita su normal desenvolvimiento social, académico, cultural, deportivo, psicológico y económico, lo que podría resumirse en un bajo nivel de Resiliencia?

\section{Desarrollo}

En 1942, los profesores Hunziker y Krapf, sostuvieron que el turismo era el conjunto de actividades y fenómenos originados por la dislocación y permanencia de personas fuera de su área habitual, siempre y cuando sus dislocaciones y permanencias no fuesen utilizadas para una actividad lucrativa principal, permanente o temporaria. En 1970 la Organización Mundial del Turismo estableció como Día Mundial del Turismo el 27 de septiembre de todos los años; sin embargo, esta actividad se ha visto afectada por factores como fluctuaciones marcadas por el tipo de cambio, la baja del precio del petróleo y de otros productos, y la creciente preocupación por la seguridad mundial.

Ante las prominentes fallas geológicas de nuestro suelo manabita, es importante establecer estrategias que permitan enfrentar acontecimientos adversos generados por la naturaleza o por el ser humano. Una de las acciones que permitirían enfrentar la grave problemática generada por un desastre, que podría ser un terremoto como el ocurrido el 16 de abril del 2016, es el turismo, ya que es una fuente de ingresos altamente rentable, que permite involucrar a cientos de autores, generando empleos y haciendo conocer las múltiples bondades del pueblo manabita. 
Las actividades turísticas son dinámicas. Durante décadas, el turismo ha experimentado un continuo crecimiento y una profunda diversificación, hasta convertirse en uno de los sectores económicos que crecen con mayor rapidez en el mundo. El turismo mundial guarda una estrecha relación con el desarrollo y se inscriben en él un número creciente de nuevos destinos. Esta dinámica ha convertido al turismo en un motor clave del progreso socio económico (Organización Mundial del Turismo, 2017). Este sector de la economía en el 2015 se vio afectado por tres factores: las fluctuaciones marcadas por el tipo de cambio, la baja del precio del petróleo y de otros productos, y la creciente preocupación por la seguridad mundial. Por este motivo, se justifica que uno de los elementos esenciales del Mandato de la Organización Mundial del Turismo (OTM) es "Ayudar al sector turístico a adoptar principios de sostenibilidad". La diversidad de pisos climáticos y las condiciones que el Ecuador ofrece a los turistas como: bosques nublados, páramos, valles, selvas tropicales en distancias cortas, lo ubican en un lugar preferido para el aventurismo. (Ministerio de Turismo, 2017), en consecuencia, a ello, Ecuador es el tercer país a escala mundial con mayor diversidad de aves; resultado arrojado en el evento internacional tercera versión Global Big Day, desarrollado el 13 de mayo del 2017, que registró 1.259 especies vistas.

El turismo para Ecuador, es uno de los sectores más importantes en el desarrollo económico y constituye una de las principales fuentes generadoras de divisas, permitiendo la generación de nuevas plazas de trabajo, nuevas inversiones locales y extranjeras, y la mejora de infraestructuras hoteleras, puertos y vías. De acuerdo al censo de vivienda del 2010, Manabí es la tercera provincia más poblada y posee el segundo puerto marítimo del país, además es privilegiada por sus 350 kilómetros de playa, desde Ayampe (en el sur) hasta Cojimíes (en el norte), geografía caracterizada por acantilados, desembocaduras, estuarios, islotes, islas, lajas y rocas que muestran bellos paisajes a lo largo de la geografía costera, siendo el principal atractivo turístico.

La poca atención a la disminución del riesgo provocado por los desastres, puede generar importantes perjuicios a la economía, así como falta de confianza en la población y en los inversores, además de perturbar drásticamente los servicios básicos de la comunidad como los sistemas de distribución de alimentos, de abastecimiento de agua, asistencia médica, transporte, eliminación de basura y comunicación, a nivel local y con el resto del mundo. Podría ocurrir que empresas e inversores privados eviten invertir en ciudades donde se perciba cierta indiferencia 
por las acciones a favor de la reducción de riesgos, en ese sentido la gestión integral u holística del riesgo de desastres es más atractiva cuando responde simultáneamente a las necesidades de muchos socios participantes y de las otras prioridades competidoras. Es decir que se requiera que la resiliencia demanda la participación de todos quienes forman parte del conglomerado mantense, donde todos deben de fortalecer la actitud para enfrentar las consecuencias de un desastre, Por lo general, los incentivos son mayores cuando la gestión de riesgos de desastres contribuye de manera visible en la mejora del bienestar económico y social. (Oficina de las Naciones Unidas para la Reducción del Riesgo de Desastres, 2012).

En un mundo globalizado que se caracteriza por la densificación de todo tipo de flujos, cobran creciente importancia las relaciones a diferentes escalas que desde las ciudades se establecen con el exterior, tanto de sentido vertical como horizontal (A.\& Rodríguez-Pose, 2011).

\section{Objetivo}

El objetivo principal, es desarrollar la estrategia que permita al ecuatoriano fortalecer de manera especial al manabita, mejorar el carácter y desarrollar habilidades y destrezas psicológicas y físicas con la resiliencia adecuada, a través de múltiples actividades, que serán parte de la rutina y del hábitat, lo que permitirá resistir, sobrevivir y desarrollar y crecer ante desastres.

Se pueden considerar actividades que involucren la innovación, lo que constituyen un elemento clave para lograr el desarrollo urbano; sin embargo, actualmente existen ciudades que, pese a ser polos científico-tecnológicos y contar con numerosas empresas innovadoras, no necesariamente traduce eso en un desarrollo más inclusivo para sus ciudadanos, más sostenible en el plano ambiental o en una gestión pública más eficaz. De ahí la necesidad de convertir también a las ciudades en ambientes socialmente innovadores. (Valle, 2013), pues, la innovación es vista como un proceso dinámico en el que el conocimiento se acumula mediante el aprendizaje y las interacciones. (Organización de Cooperación y Desarrollo Económicos, 2005)

La globalización de productos y mercados, tiene tres implicaciones importantes para la competencia: las fronteras de la industria no son las mismas que las fronteras nacionales, porque muchas industrias se están volviendo globales; el cambio de los mercados nacionales a los globales ha intensificado la rivalidad competitiva entre industria; los oligopolios que alguna vez dominaron el mercado con tres o cuatro empresas al frente con competencia mínima extranjera, 
se han fragmentado y muchas otras luchan por una parte de su mercado. Aunque la globalización ha incrementado la amenaza al ingreso como la intensidad de la rivalidad entre muchos mercados locales protegidos, también ha creado enormes oportunidades para los participantes de esos mercados (Oliver, 2012). Ejemplo de ello es la provincia de Manabí, que a pesar de terremoto del 16 de abril del 2016 se está levantando. En Manta el 12 de mayo se inauguró el Sail Plaza Hotel, esta infraestructura tiene una construcción de 37.500 metros cuadrados y demandó de una inversión de 51 millones de dólares, de los cuales el $60 \%$ fue financiado por la Corporación Financiera Nacional (CFN) y el $40 \%$ restante fue inversión privada mantense.

Además, el 20 de abril se abrió el Mall del Pacífico, con 170 locales comerciales, 1500 parqueaderos, la inversión es de 200 millones de dólares y dará trabajo a unas 1500 personas, ayudando de esta manera a la economía de la provincia.

El factor geopolítico influye en el desarrollo de ciudades, pues lo que ocurre en un lugar del planeta afecta todo el resto, donde la relación que tiene el hombre con la sociedad es importante. En virtud de lo cual, y conociendo que la economía es global, las empresas deben conocer lo que está pasando en el mundo para enfrentar retos, y prever lo que sucederá en los próximos años.

\section{Método}

La presente investigación utiliza una metodología con enfoque cualitativo de carácter descriptivo, tomando como referencia conceptos de competitividad, turismo, resiliencia, marketing holístico, el análisis determina el nivel de recuperación de la provincia de Manabí principalmente en unos de los sectores de la economía, como es el turismo, afectado por el desastre natural del 16 de abril de 2016. Se consideran datos proporcionados por el Instituto Nacional de Estadísticas y Censos (INEC), el Banco Central del Ecuador (BCE), Oficina para la Reducción de Riesgos de Desastres de las Naciones Unidas (UNISDR), Organización Mundial del Turismo (OTM), Objetivos de Desarrollo Sostenible de la ONU y con apreciaciones propias de los autores, corroboradas por opiniones especializadas difundidas sobre el tema. 


\section{Encuestas:}

1.- Considera Ud., que aún existen secuelas psicológicas del terremoto del 16 de abril del 2016, que afectan el normal desenvolvimiento en el cantón de San Pablo de Manta.

\begin{tabular}{|l|r|r|}
\hline \multirow{2}{*}{\multicolumn{1}{|c|}{ RESPUESTA }} & \multicolumn{2}{c|}{ TOTAL } \\
\cline { 2 - 3 } & CANTIDAD & \multicolumn{1}{c|}{$\%$} \\
\hline SI & 14 & 66,67 \\
\hline NO & 1 & 4,76 \\
\hline NO LO SÉ & 6 & 28,57 \\
\hline TOTAL & 21 & 100,00 \\
\hline
\end{tabular}

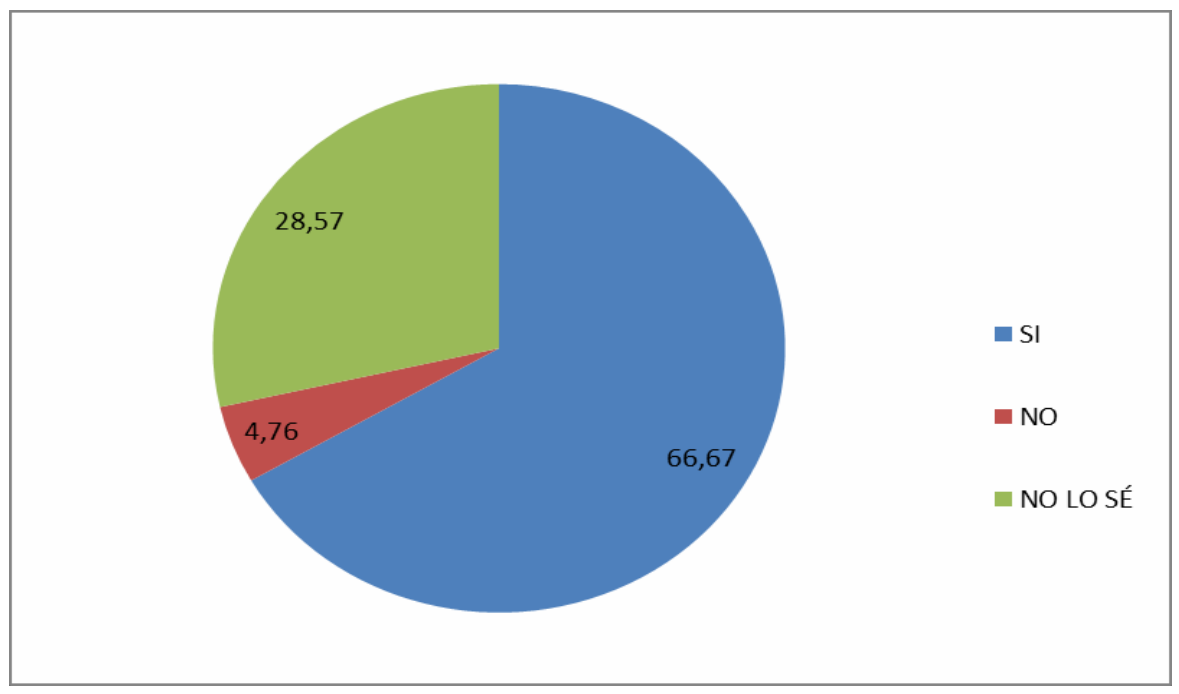

2.- ¿Piensa que se repetirá un terremoto similar al 16 a?

\begin{tabular}{|l|r|r|}
\hline \multirow{2}{*}{\multicolumn{1}{|c|}{ RESPUESTA }} & \multicolumn{2}{c|}{ TOTAL } \\
\cline { 2 - 3 } & CANTIDAD & \multicolumn{1}{c|}{$\%$} \\
\hline SI & 7 & 33,33 \\
\hline NO & 0 & 0 \\
\hline NO LO SÉ & 14 & 66,67 \\
\hline TOTAL & 21 & 100,00 \\
\hline
\end{tabular}




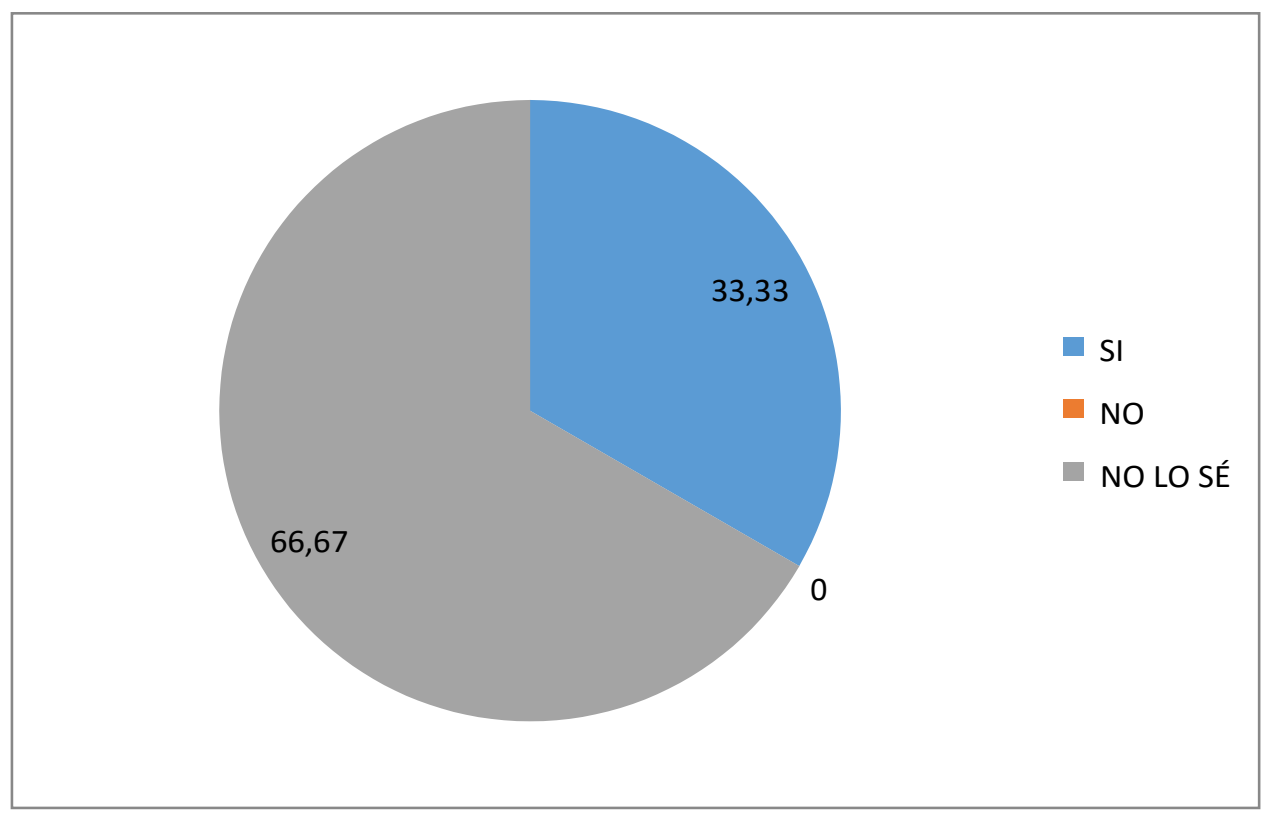

3.- Conoce Ud., ¿Qué es la Resiliencia?

\begin{tabular}{|l|r|r|}
\hline \multirow{2}{*}{\multicolumn{1}{c|}{ RESPUESTA }} & \multicolumn{2}{c|}{ TOTAL } \\
\cline { 2 - 3 } & CANTIDAD & \% \\
\hline SI & 6 & 28,57 \\
\hline NO & 15 & 71,43 \\
\hline TOTAL & 21 & 100,00 \\
\hline
\end{tabular}

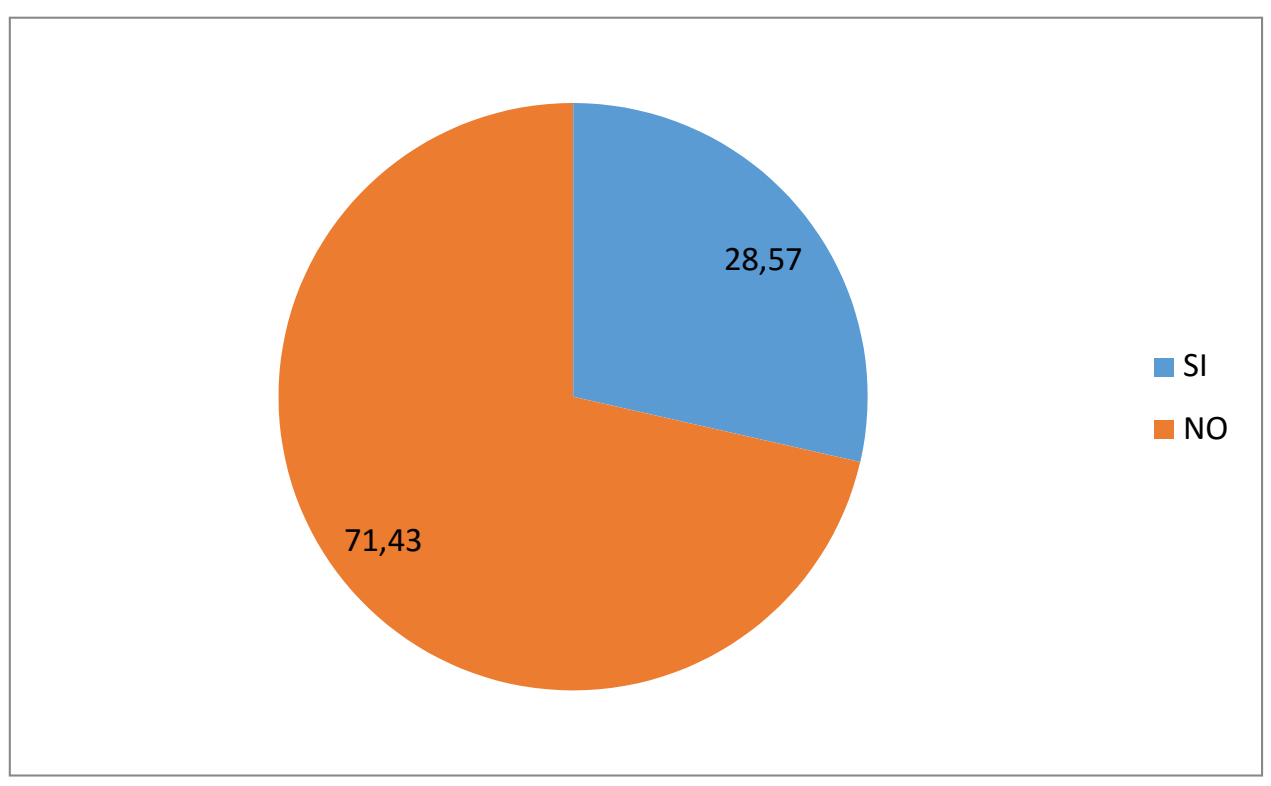


4.- ¿Está de acuerdo que la cultura de riesgo sea una materia que se incluya en la malla curricular de todas las carreras?

\begin{tabular}{|c|c|c|}
\hline \multirow[t]{2}{*}{ RESPUESTA } & \multicolumn{2}{|c|}{ TOTAL } \\
\hline & CANTIDAD & $\%$ \\
\hline SI & 21 & 100 \\
\hline $\mathrm{NO}$ & 0 & 0 \\
\hline TOTAL & 21 & 100 \\
\hline
\end{tabular}

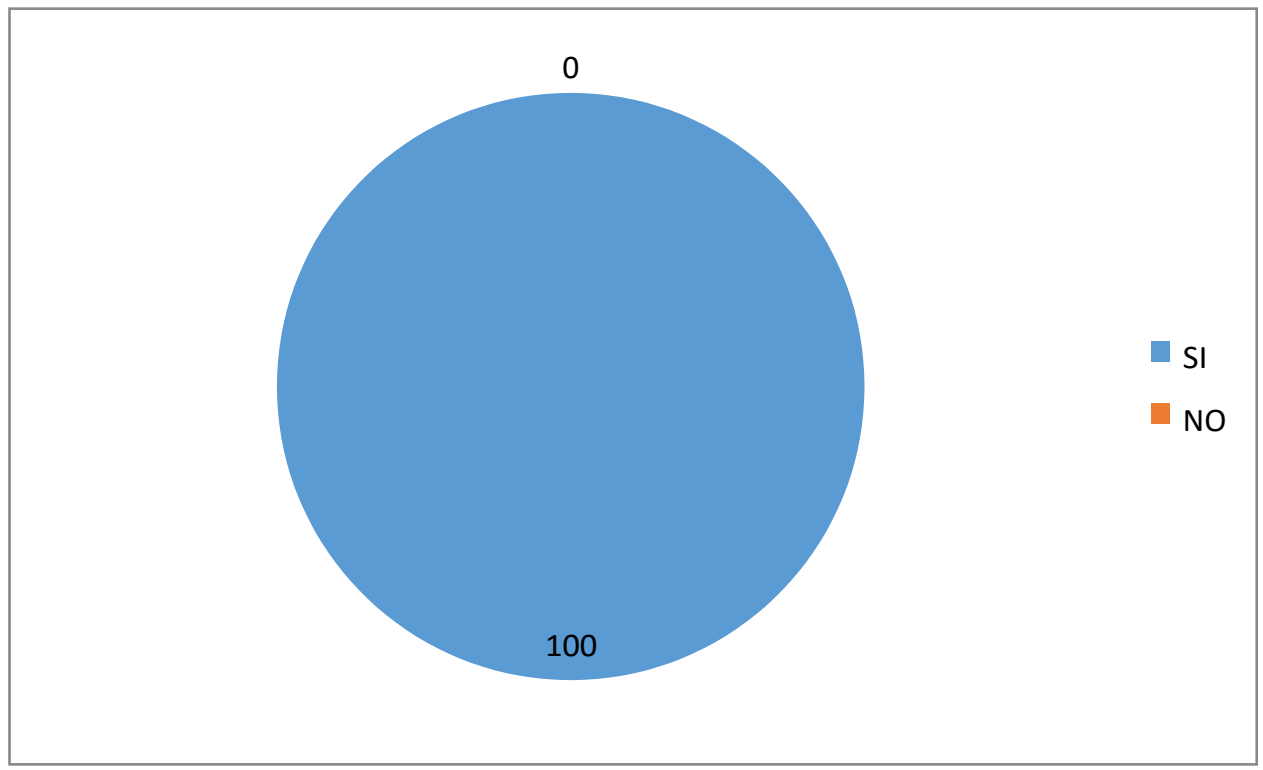

5.- Está de acuerdo que existan actividades para fortalecer la resiliencia en Manta?

\begin{tabular}{|l|r|r|}
\hline \multirow{2}{*}{\multicolumn{1}{|c|}{ RESPUESTA }} & \multicolumn{2}{c|}{ TOTAL } \\
\cline { 2 - 3 } & \multicolumn{1}{c|}{ CANTIDAD } & \multicolumn{1}{c|}{} \\
\hline SI & 21 & 100 \\
\hline NO & 0 & 0 \\
\hline TOTAL & 21 & 100 \\
\hline
\end{tabular}




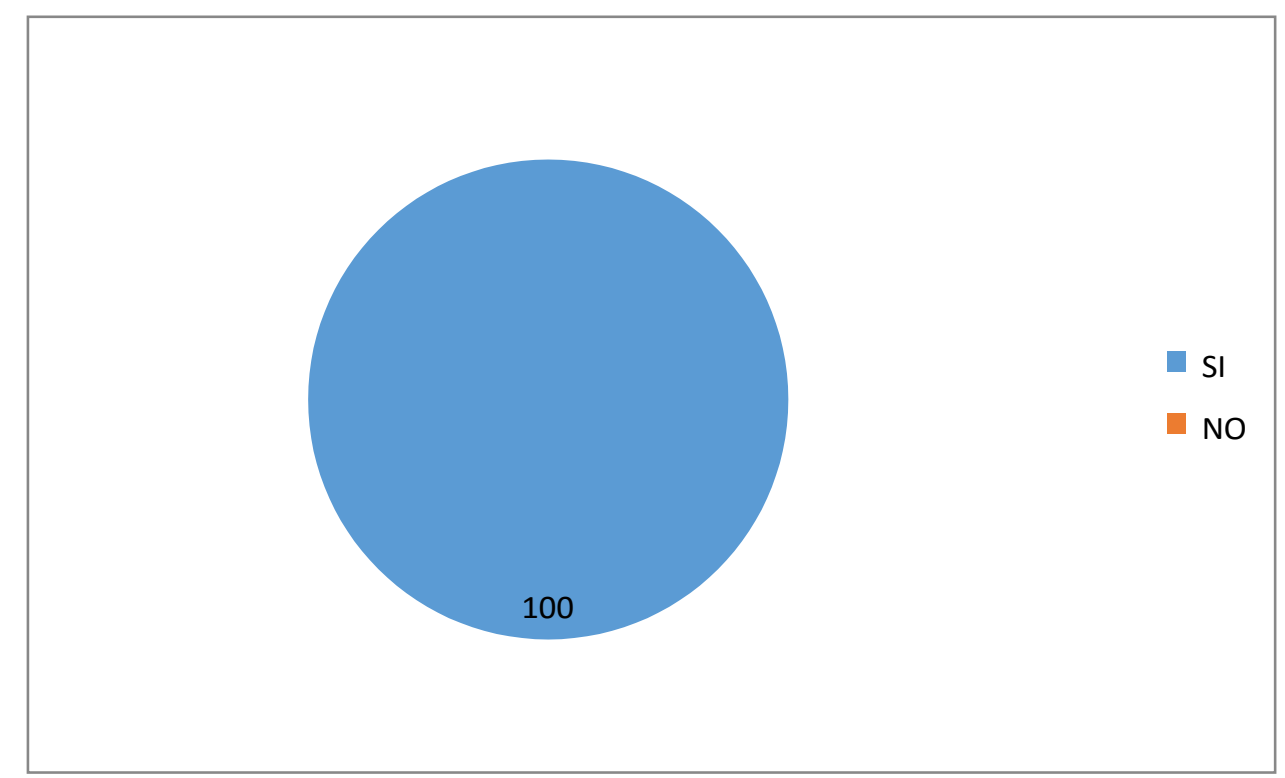

Desde una perspectiva holística para un desarrollo sostenible, sensible al riesgo de desastre, se puede lograr un menor impacto y pérdidas a consecuencia de las amenazas naturales; por ejemplo, la Campaña Mundial "Desarrollando ciudades resilientes - !Mi ciudad se está preparando!"” de la Oficina para la Reducción de Riesgos de Desastres de las Naciones Unidas, a la que más de 3000 ciudades en el mundo se han adherido, de las cuales, 1400 corresponden a ciudades y gobiernos locales del continente americano (América Latina, el Caribe y Norteamérica).

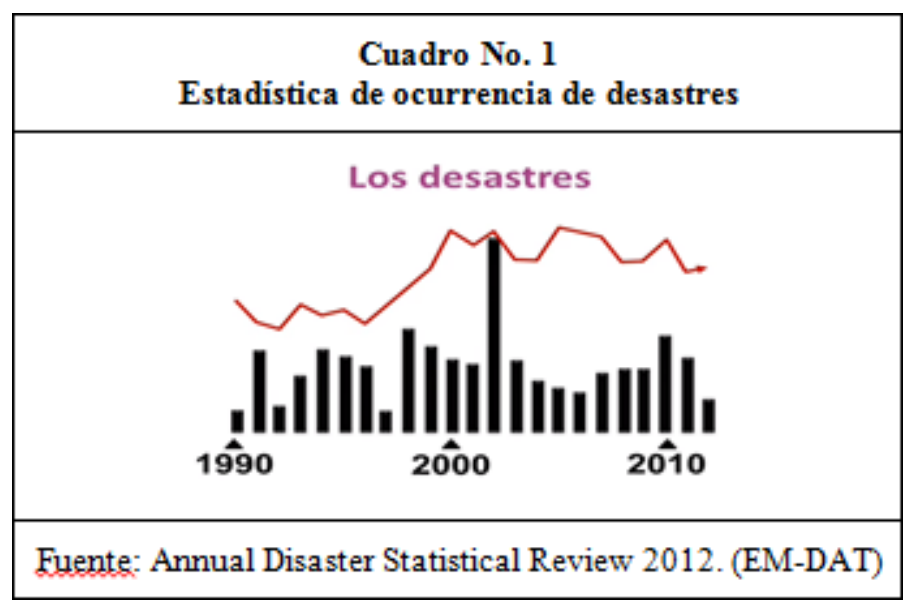


Los desastres han perjudicado a 4.400 millones de personas en el mundo, ocasionando daños que ascienden a más de 2 billones de dólares americanos, y cobrando la vida de 1.3 millones de personas (Luis Manuel García Moreno, Secretario de Protección Civil - Taller de Campaña Mundial Desarrollando Ciudades Resilientes, desarrollado en Chiapas).

De acuerdo a datos proporcionados por la UNISDR, en el año de 1913 el 10\% de la población vivía en las ciudades, en el 2013 el 50\% y para el año 2075 el porcentaje alcanzará un 75\% de personas. Las ciudades dependiendo de sus desplazamiento y desarrollo pueden estar expuestas a múltiples amenazas: Sismos, Erupciones volcánicas, Tsunamis, Movimientos de laderas, Incendios forestales, Olas de Frío, Olas de Calor y las creadas por el hombre como: pandemias, contaminación, incendios urbanos y forestales, entre otras.

El 16 de abril del 2016, un terremoto masivo de magnitud 7,8 en la escala de Richter sacudió la costa noroeste del Ecuador, el mismo que además de ocasionar grandes pérdidas humanas y materiales, agravó factores preexistentes de vulnerabilidad social y económica en Esmeraldas y Manabí, las mismas que ya venían presentando situaciones complejas de pobreza en 2014 (Esmeraldas 43,2\% y Manabí 31,3\%) y de ruralidad en 2010 (Esmeraldas 41,7\% y Manabí 41,0\%), según el Instituto Nacional de Estadística y Censos (INEC), donde adicionalmente gran cantidad de viviendas no contaban con acceso a redes de agua (43,4\% en Esmeraldas y 49,1\% en Manabí) donde el acceso a saneamiento con alcantarillado representaba el 30,7\% y 33,3\%, respectivamente. (Instituto Nacional de Estadística y Censo, 2010).

A seis meses de ocurrido el evento natural, Reliefweb efectuó un balance de lo ocurrido, estimando que más de 300.000 personas aún requieren de una vivienda segura, observando una reducción del número de personas ocupando albergues o sitios espontáneos de 30.000 al principio de la emergencia a 16.000 aproximadamente. Las pérdidas económicas producto del terremoto repercuten en la generación de empleos y en la forma de vida de las ciudades afectadas y por ende en la economía nacional. De acuerdo a la información de la Secretaría Nacional de Planificación y Desarrollo (SENPLADE), el desastre natural suscitado provoca un impacto negativo del $0.7 \%$ en el PIB del país y una reducción de 21.823 plazas de trabajo en 2016. Tras el terremoto, el Estado creó el Registro Único de Damnificados (RUD) a fin de identificar personas afectadas y brindarles apoyo. Hasta el 7 de octubre, el Estado verificó y oficialmente reconoció a 
386.985 personas en el RUD (Ministerio Coordinador de Desarrollo Social - MCDS, 7 octubre). (Reliefweb, 2016)

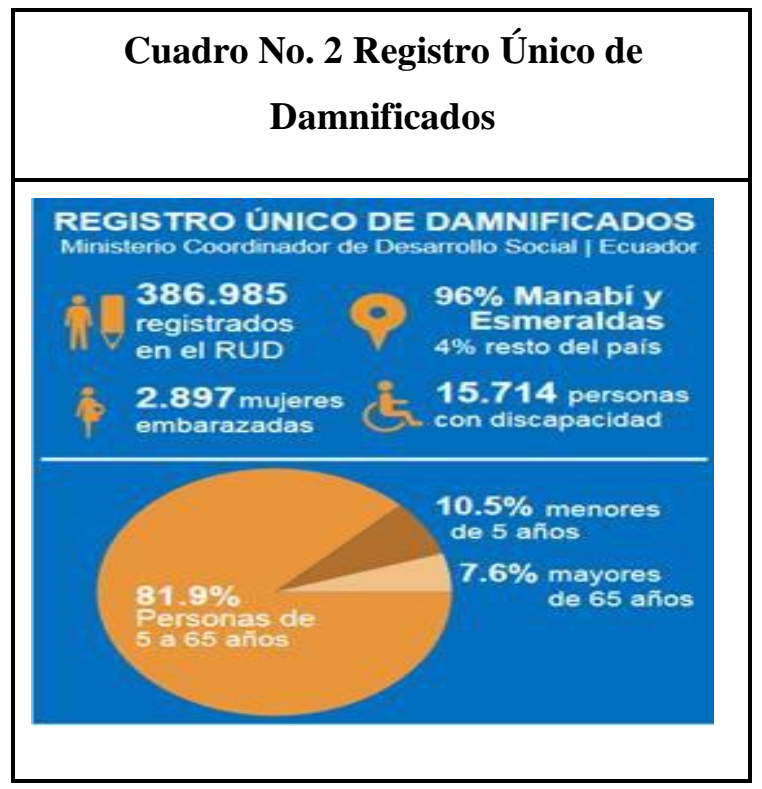

El terremoto del 16 de abril del 2016 provocó un fenómeno de comportamiento social importante, donde los habitantes de las provincias afectadas (Manabí y Esmeraldas) reaccionaron con una gran fuerza de voluntad social frente al terremoto, generando una actitud de reconstrucción. A pesar de la infraestructura de ciudad, que no era la más idónea, los turistas nacionales no dudaron en visitar a las provincias afectadas, ayudando de esta manera a reactivar y fortalecer la economía.

En la reactivación de Manta el Gobierno Autónomo Descentralizado (GAD) tuvo un importante apoyo por parte del Gobierno Nacional en la reubicación de 1.700 comerciantes de Tarqui, de los cuales se ha capacitado aproximadamente a 700 en la reactivación, manejo y reposicionamiento de sus negocios, donde más de 120 participantes son mujeres emprendedoras. Adicionalmente, 166 locales afectados fueron reparados para reactivar la actividad económica, y se rehabilitó la infraestructura productiva de 312 productores con sus familias quienes gracias a esto recuperaron sus medios de vida. Es imperante efectuar un proceso de recuperación post-terremoto a mediano y largo plazo, el cual debe considerar un desarrollo económico inclusivo para lograr un crecimiento local, que involucre un trabajo eficiente para el desarrollo del comercio, turismo, 
acuacultura, pesca y agricultura, considerando a los grupos de atención prioritaria y en base a los criterios de reconstruir mejor y de forma sostenible. (Reliefweb, 2016)

Los impactos de un desastre pueden ser devastadores, por lo tanto, la resiliencia hace referencia a la resistencia y capacidad de los seres humanos para soportar cambios inesperados y poder transformar formas de vida y levantarse. Es ahí donde las universidades no pueden mantenerse aisladas; pues en el mundo académico se reconocen los umbrales de cambio y aproximaciones del conocimiento, para dar respuestas a las necesidades de la población.

Por lo tanto, en la planificación estratégica, los gobiernos deben considerar la proyección de crecimiento de habitantes en las ciudades y aunar esfuerzos para lograr una innovación económica, la competitividad en la producción y comercialización de bienes y servicios, con el fin de aumentar la oferta laboral, calidad de vida de sus habitantes y garantizar un urbanismo sostenible.

El marketing holístico visto como la integración de las estrategias de marketing, la tecnología, la conciencia de la empresa hacia la orientación al cliente, es definitivamente el arma con la que pueden luchar las empresas por crear reconocimiento y sobresalir en un mercado globalizado y sumamente competitivo. (Sarnari, 2013)

La aplicación responsable de los fundamentos del marketing holístico en las diferentes actividades comerciales de bienes y servicios, ayudará a desarrollar la resiliencia, y la competitividad para alcanzar el desarrollo económico de la localidad. Sin embargo, a pesar de la importancia de contar con información sobre los desastres, existen personas que desconocen el impacto climático y sus efectos que repercuten sobre la sociedad.

El principio de la Animación Turística, como estrategia pedagógica a la que se le puede adicionar un marco socio-ecológico, tiene todo el potencial para convertirse en el hilo conductor de una reconstrucción basada en una serie de factores resilientes, siendo uno de los elementos esenciales del Mandato de la Organización Mundial del Turismo (OTM), "Ayudar al sector turístico a adoptar principios de sostenibilidad", se determinó que los factores motivantes de aplicación general al turismo detectados como más importantes son: la educación de su habitantes, la urbanización, la aplicación de un marketing responsable, las agencias de viajes y las atracciones de los destinos. 
La ubicación geográfica de Manabí constituye uno de los factores más importantes para su desarrollo turístico, motivo por el cual es considerada una provincia con un gran atractivo turístico en la región costera, donde sus paisajes, playas, gastronomía y calidez de su gente, la convierten en uno de los lugares preferidos por los turistas nacionales y extranjeros. De acuerdo a datos proporcionados por el departamento de turismo, en el 2014 arribaron a Manta más de 500.000 turistas. Al analizar la demanda turística de Ecuador y de forma específica de Manabí; este es influenciado por los impactos del cambio climático en el planeta, elementos vulnerables (población, infraestructura, medio ambiente), donde es necesario plantear y generar procesos sociales de adaptación y resiliencia que permitan dar atención a la disminución del riesgo, adaptando sistema de abastecimiento de alimentos, agua, transporte, atención médica, comunicación, etc.

\section{Resultados}

1. Existe un elevado número de manabitas, que aún recuerdan con nostalgia y preocupación, la tragedia ocurrida el 16 de abril del 2016, cuyos resultados influyen en el desenvolvimiento y comportamiento de este número de personas, ya que temen que hechos similares se repitan, pudiendo citar ejemplos de personas que evitan vivir y estar en lugares altos, como edificaciones.

2. De igual forma se puede establecer que la Cultura de Riesgos es de muy poco conocimiento a nivel general, minimizando en su real magnitud, la importancia e incidencia que esto implica, ya que su aplicación, mejoraría la capacidad de resistencia y reacción ante los desastres.

3. En los resultados se pueden apreciar la aportación y participación gubernamental es muy irrisoria, alejándose del cumplimiento de los requerimientos existentes.

4. Empleando los descriptores «resilience $\ll O R » p s y c h o l o g i c a l$ fitness $« O R ~ » m e n t a l$ strength $«$ como palabras clave en PsycINFO, se encontraron 8838 registros. Para restringir la búsqueda a contextos militares, se añadieron los descriptores «military» $\mathrm{OR}$ «soldier» a la primera, conectados por el operador AND, localizando 295 publicaciones. De éstas, 223 eran artículos de revistas científicas, 58 eran libros y 14 eran tesis doctorales. En cuanto a la metodología, 175 eran estudios empíricos, 151 estudios cuantitativos, 27 estudios longitudinales, 25 
entrevistas y 14 estudios prospectivos. Siguiendo con la descripción de la metodología basada en la evidencia de Castro y Adler, se realizó una tercera búsqueda añadiendo el descriptor «randomized controlled trial» a la última, de la que surgieron 40 publicaciones, todas ellas realizadas en revistas científicas y, de las cuales, 4 pertenecían al área de «salud y servicios de salud mental», 6 al área de «psicofarmacología», 6 al de «trastornos de ansiedad y neurosis» y 7 al de «terapias cognitivas». En la base de datos DTIC se encontraron 333 publicaciones. Del análisis de documentos reportados por PsycINFO y DTIC, se seleccionaron 60 documentos para hacer la revisión y 145 para hacer el artículo.

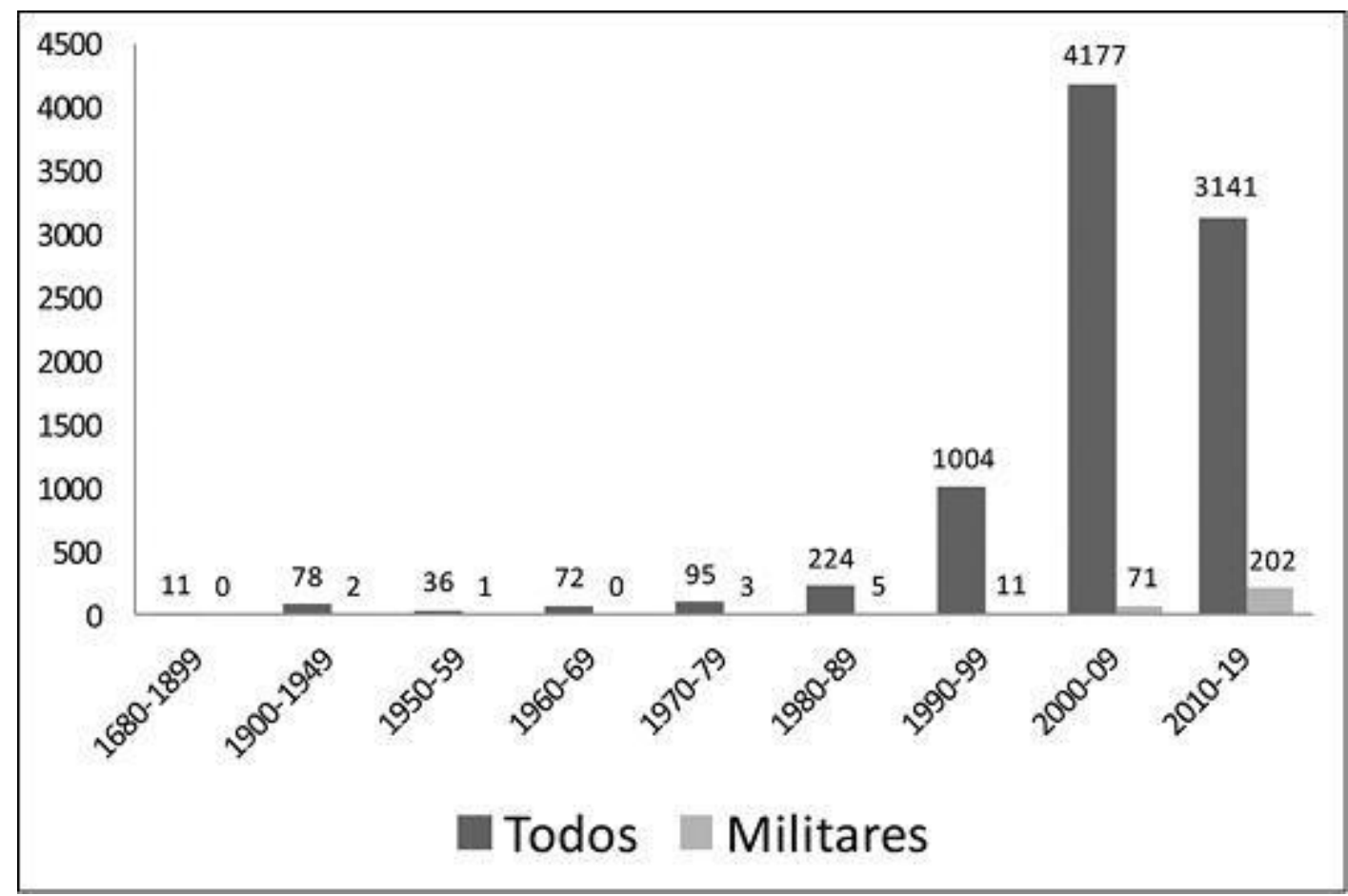

Figura 1. Evolución de las publicaciones sobre resiliencia en la comunidad científica.

Fuente: PsycINFO (03/07/2013).

\section{Discusión}

Al confrontar los factores que intervienen, es notoria que amerita de manera urgente, la participación decidida de todos los niveles de estado: Político Estratégico, Estratégico, Operacional y Táctico. 
Nivel Político Estratégico, es el nivel estrictamente de decisión, donde a nivel jefatura de estado del gobierno se establece que hacer o no ante cualquier eventualidad, escenario y lugar geográfico del país, siendo el presidente el representante legal quien toma la decisión del estado.

Nivel Estratégico: es el ente planificador que pone a consideración al nivel superior todos los cálculos, acciones, presupuestos, y consideraciones estratégicas en beneficio de solucionar un problema o fortalecer la misión administrativa de un gobernante. En nuestro país los niveles de planificación superior se encuentran en los ministerios y Gobiernos Autónomos y Descentralizados de los cantones.

Nivel Operacional: son los niveles de mandos medios que intervienen en la ejecución de disposiciones del nivel superior con todas las directrices establecidas.\}

Nivel Táctico; son todos los ejecutores de la planificación estratégica a fin de estar en contacto cercano con los usuarios.

Todos estos niveles son necesarios establecerlos a fin de clarificar las diferentes etapas de los procesos, los que para el presente trabajo permitirá fortalecer y cumplir los objetivos de manera organizada en todas las etapas del proceso.

Es relevante denotar la participación activa de todos los actores, con el fin de alcanzar el objetivo general y los específicos, donde cada uno son eslabones importantes de la cadena de resiliencia necesaria para enfrentar catástrofes naturales o creadas por el hombre, en el caso particular de Manta, se requiere mayor atención gubernamental (el estado) y seccional (municipio), así como la participación de todas las instituciones públicas y privadas y de cada uno de los ciudadanos manabitas y mantenses, a fin de multiplicar los esfuerzos en todas las actividades planteadas, como charlas y conferencias de capacitación periódicas, simulacros, creación de infraestructura que permitan centralizar todas las actividades.

Las actividades turísticas deben ser de primer orden, para atender de mejor manera la visita de los cruceros, así como creación de emprendimientos con lugares recreacionales, que irán en beneficio del turismo, fuente necesaria para que Manta sea la fuente de ingreso principal de la provincia de Manabí. 
De acuerdo al ministerio de turismo, las estadísticas de ingreso por ámbito de comercio y turismo han ido creciendo de manera sostenida y con interesante proyección, lo que proyecta mejores días en lo social, psicológico y económico para el cantón de San Pablo de Manta.

\section{Conclusiones}

Luego del análisis efectuado se puede establecer las siguientes conclusiones:

1.- Definitivamente el desastre ocurrido el 16 A del 2016 afectó, afecta y afectará el normal desenvolvimiento del ciudadano mantense y manabita; de acuerdo a las encuestas realizadas muchos son los que presentan resistencia al cambio y mejoras.

2.- No existen suficientes espacios económicos que permitan fortalecer las actividades productivas de la provincia, donde el gobierno nacional, provincial y local, trabajen mancomunadamente en equipo, favoreciendo a la democracia local y participativa, ya que el grado de Resiliencia se encuentra por debajo del nivel requerido permitiendo mejorar la calidad de vida de sus habitantes.

3.- No existe la infraestructura para el trabajo en un modelo sistémico para atender de forma específica necesidades de la población que ayude a posicionar la producción local, mediante una planificación estructurada y a la optimización de procesos en la consecución de metas, para satisfacer y mejorar la calidad de vida en el entorno socioeconómico en que se desenvuelven sus habitantes.

4.- La resiliencia y la reducción del riesgo de desastres deben formar parte del diseño y estrategias urbanas para lograr un desarrollo sostenible. Los desastres no son obras de la naturaleza, son problemas del desarrollo, un desarrollo mal planteado, donde los gobiernos locales son los llamados a trabajar, por lo que se debe motivarlos y que se comprometan a efectuar acciones que ellos desarrollan en los territorios, a efectos de que se puedan mitigar y dar paso a la resiliencia de los territorios.

Para concluir, se emula lo referente en el documento oficial de la Organización de las Naciones Unidas, denominado: "Guía de los Vagos para salvar al mundo", resaltando lo siguiente: 
“Acabar con la pobreza extrema. Luchar contra la desigualdad y la injusticia. Solucionar el problema del cambio climático. Vaya. Los objetivos mundiales son importantes, son objetivos que cambiarán el mundo y para los que será necesaria la cooperación entre los gobiernos, las organizaciones internacionales y los líderes mundiales. Parece imposible que las personas de a pie puedan influir en algo. ¿Va a rendirse sin más?”

“¡No! El cambio empieza por usted. De verdad. Todos y cada uno de los seres humanos del mundo, hasta los más indiferentes y perezosos, forman parte de la solución. Por suerte, hay cosas facilísimas que podemos introducir en nuestra rutina y, si todos lo hacemos, lograremos grandes cambios". (Organización de las Naciones Unidas, 2017)

El individuo es capaz de afrontar cambios, sumar esfuerzos para desarrollar una resiliencia justa, equitativa, sostenible; prestar atención a estos conceptos, ayudará a fortalecer procesos y capacidades, para que las ciudades puedan resistir crisis, tanto humanas como naturales.

El Marketing Holístico aplicado al desarrollo comercial, permitirá estudiar las necesidades de los consumidores, para que de forma sostenible y sustentable se responda a los requerimientos de los individuos y las ciudades o países logren ser competitivos tanto en el mercado local como global.

\section{Referencias bibliográficas}

A., \& Rodríguez-Pose, A. y. (2011). Handbook of local and regional development. Nueva York: Routledge, 413-24.

Cicchetti, D. y. (1993). Prospects and promises in the study of resilience. Development and Psychopathology., 497-502.

Herrera, W. (26 de 04 de 2016). Diario El Comercio. Obtenido de http://www.elcomercio.com/opinion/ciudadesresilientes-terremoto-reconstruccion.htm

Ministerio de Turismo. (31 de 05 de 2017). Ecuador, tercer país con mayor diversidad de aves en el mundo. Obtenido de http://www.turismo.gob.ec/ecuador-tercer-pais-con-mayor-diversidad-deaves-en-el-mundo/

Instituto Nacional de Estadística y Censo (INEC). (30 de 05 de 2010). Pobreza por Ingresos. Obtenido de http://www.ecuadorencifras.gob.ec/pobreza-por-ingresos/ 
Oficina de las Naciones Unidas para la Reducción del Riesgo de Desastres (UNISDR). (2012). Cómo desarrollar ciudades más resilientes. Ginebra: United Nations.

Oliver, P. (06 de 04 de 2012). Estrategia Empresarial. Obtenido de http://estrategiaoutsourcingolivierperez.blogspot.com/2012/04/estrategia-en-el-entornoglobal_06.html

Organización de Cooperación y Desarrollo Económicos OCDE y Oficina de Estadísticas de las Comunidades Europeas Eurostat. (2005). Manual de Oslo, Guía para la recogida e interpretación de datos sobre innovación, OECD/European Communities. España: Grupo TRAGSA $3^{\text {a }}$ edición. Organización de las Naciones Unidas. (26 de 05 de 2017). Objetivos de Desarrollo Sostenible de la ONU. Obtenido de http://www.un.org/sustainabledevelopment/es/takeaction/\#

OTM. (26 de 05 de 2017). Organización Mundial del Turismo. Obtenido de http://www2.unwto.org/es/content/por-que-el-turismo.

Sarnari, G. (18 de 01 de 2013). marketing-holistico-social-y-relacional. Obtenido de https://gustavosarnarimkt.wordpress.com

Santos, R. (2013). Levantarse y luchar: Cómo superar la adversidad con la resiliencia. España: Penguin Random House.

Reliefweb. (13 de 10 de 2016). Reliefweb. Obtenido de http://reliefweb.int/report/ecuador/ecuador-terremoto-informe-seis-meses-13-de-octubre-2016 Valle, R. M. (2013). Estrategias de innovación para el desarrollo y la resiliencia de ciudades medias. Documents d'Anàlisi Geogràfica 2013, vol. 59/3 481-499, 481-499. 


\section{Anexos}

Remembranzas del terremoto del 16 de abril del 2016
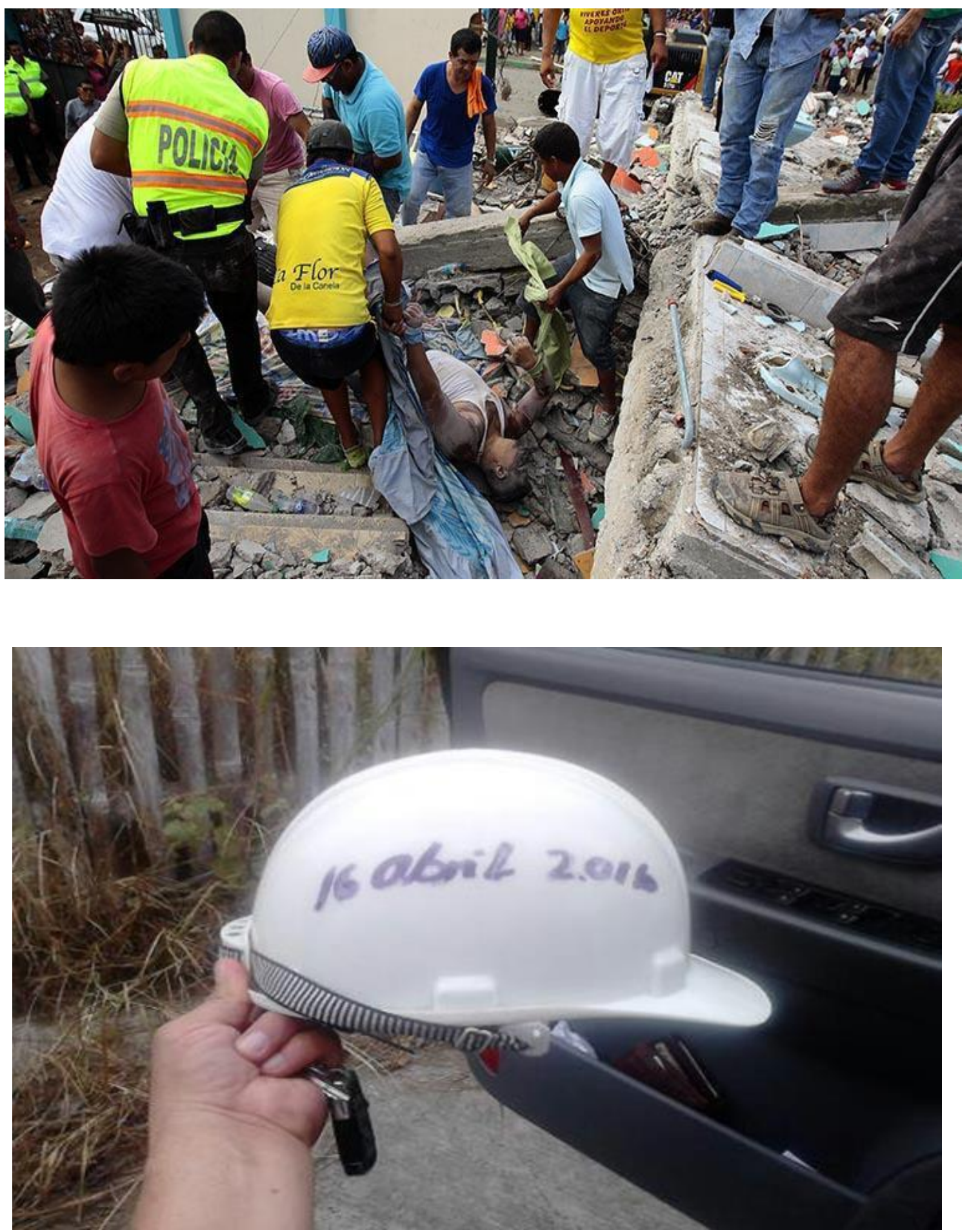

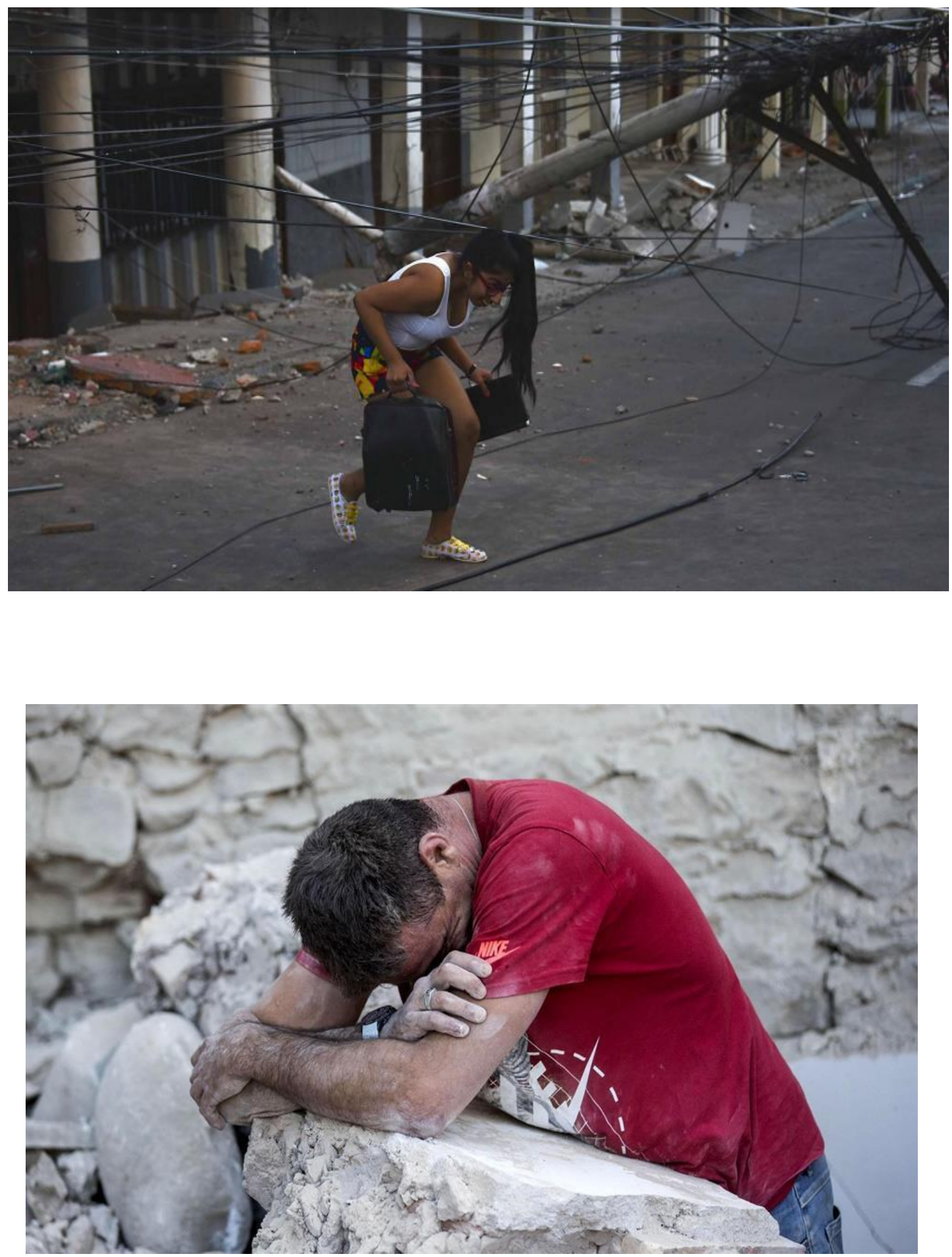